Research Article

\title{
Some Existence and Stability Criteria to a Generalized FBVP Having Fractional Composite $p$-Laplacian Operator
}

\author{
Sh. Rezapour $\mathbb{D}^{1,2}$ S. T. M. Thabet $\mathbb{D}^{1,}{ }^{3}$ M. M. Matar $\mathbb{D},{ }^{4}$ J. Alzabut $\mathbb{D}{ }^{5,6}$ and S. Etemad $\mathbb{D}^{1}$ \\ ${ }^{1}$ Department of Mathematics, Azarbaijan Shahid Madani University, Tabriz, Iran \\ ${ }^{2}$ Department of Medical Research, China Medical University Hospital, China Medical University, Taichung, Taiwan \\ ${ }^{3}$ Department of Mathematics, University of Aden, Aden, Yemen \\ ${ }^{4}$ Department of Mathematics, Al-Azhar University-Gaza, Gaza, State of Palestine \\ ${ }^{5}$ Department of Mathematics and General Sciences, Prince Sultan University, Riyadh 11586, Saudi Arabia \\ ${ }^{6}$ Group of Mathematics, Faculty of Engineering, Ostim Technical University, Ankara 06374, Turkey
}

Correspondence should be addressed to S. Etemad; sina.etemad@azaruniv.ac.ir

Received 11 May 2021; Accepted 10 October 2021; Published 25 October 2021

Academic Editor: Liliana Guran

Copyright (c) 2021 Sh. Rezapour et al. This is an open access article distributed under the Creative Commons Attribution License, which permits unrestricted use, distribution, and reproduction in any medium, provided the original work is properly cited.

In this paper, we consider a generalized Caputo boundary value problem of fractional differential equation with composite $p$ Laplacian operator. Boundary value conditions of this problem are of three-point integral type. First, we obtain Green's function in relation to the proposed fractional boundary value problem and then for establishing the existence and uniqueness results, we use topological degree theory and Banach contraction principle. Further, we consider a stability analysis of UlamHyers and Ulam-Hyers-Rassias type. To examine the validity of theoretical results, we provide an illustrative example.

\section{Introduction}

Fractional calculus, as a generalization of classical ordinary calculus to integrodifferential operators in the noninteger settings, has attracted considerable interest in recent years and has grown rapidly since its introduction. Fractional calculus is now broadly used in several fields such as biology, fluid dynamics, viscoelastic theory, neural networks, and epidemic models; see for instance $[1,2]$ and references therein.

By using fixed point techniques, a large number of researchers studied the existence-uniqueness properties of solutions for different classes of differential equations in the fractional settings. In 2016, Ntouyas et al. [3] studied two fractional boundary value problems (FBVPs) with three-point boundary conditions and derived the existence results by using the fixed point notion. Similarly, in [4], Boutiara et al. used fixed point theorems to prove the existence results for another FBVP with three-point boundary conditions in the context of the Caputo-Hadamard and Hadamard operators. More recently, Derbazi et al. [5] designed a new FBVP by applying the generalized $\psi$-opera- tors and proved their desired results via monotone iterative techniques.

As you know, every numerical method must be accurate in order to give desired results which are acceptable for different applications. For this purpose, the analysis of the stability is needed. Various types of stability involving exponential, Lyapunov, and Mittag-Leffler have been studied for different types of problems. The abovementioned types of stability have been improved for many differential equations in both linear and nonlinear fractional cases and their related systems over the last few years. However, the stability of some nonlinear systems undergo unavoidable deficiencies which appear due to the need of predefining Lyapunov function. This is often considered as an uneasy task.

In $[6,7]$, Ulam and Hyers have initiated the concept of Ulam-Hyers stability. In addition, this notion has been considered for nonlinear fractional differential equations and their related systems. For instance, Abdo et al. [8] investigated the stability criteria for $\psi$-Hilfer fractional integrodifferential equations and in the same time, Zada et al. [9] derived similar results for impulsive integrodifferential 
equations with Riemann-Liouville boundary conditions. In [10], Kheiryan and Rezapour considered a new multisingular FBVP and checked its Hyers-Ulam stability.

On the other hand, some properties of solutions of FBVPs including the uniqueness, existence, and stability notions have been investigated with the help of various techniques such as topological degree theory (T-degree theory) and fixed point theory. In this paper, we will apply the existing concepts in $\mathrm{T}$-degree theory, as well as there are a large number of nonlinear mathematical models in engineering and the scientific fields to investigate and analyze dynamical systems. One of the most important nonlinear operators frequently used is the classical $p$-Laplacian operator. Models with $p$-Laplacian operators are often used to simulate practical problems such as tides caused by celestial gravity and elastic deformation of beams. Such extensive applications attract the attention of many researchers to study mathematical models having $p$-Laplacian operators.

Specifically, Ma et al. [11] defined a new multipoint FBVP with p-Laplacian operator and derived the existence and iteration of monotone positive solutions for the given system. Next, Matar et al. [12] studied another $p$-Laplacian FBVP having Caputo-katugampula fractional derivatives recently. For more details about $p$-Laplacian fractional boundary value problems, we refer to $[13,14]$.

Also, to see the importance of existing techniques in Tdegree theory, we can point out to a paper published by Shah and Khan [15] on the existence-uniqueness results to a coupled system of FBVPs. Further, Sher et al. [16] implemented a qualitative analysis on a multiterm delay FBVP with the help of the same technique in T-degree theory.

In 2017, Ali et al. [17] studied a coupled fractional structure of a system involving two differential equations with non-integer boundary conditions of integral type which takes the form

$$
\begin{cases}{ }^{C} D_{0^{+}}^{r_{1}} v_{1}(t)=\phi_{1}\left(t, v_{2}(t)\right), & \leq t \leq 1, \\ { }^{C} D_{0^{+}}^{r_{1}} v_{1}(t)=\phi_{2}\left(t, v_{1}(t)\right), & \leq t \leq 1, \\ v_{1}(0)=0, & v_{1}(1)=\frac{1}{\Gamma(\alpha)} \int_{0}^{K}(K-s)^{\alpha-1} p\left(v_{1}(s)\right) d s, \\ v_{2}(0)=0, & v_{2}(1)=\frac{1}{\Gamma(\beta)} \int_{0}^{K}(K-s)^{\beta-1} p\left(v_{2}(s)\right) d s,\end{cases}
$$

where ${ }^{C} D_{0^{+}}^{r_{1}}(\cdot),{ }^{C} D_{0^{+}}^{r_{2}}(\cdot)$ stands for the Caputo derivative of orders $1<r_{1}<2$ and $1<r_{2}<2$, respectively, and $\phi_{1}, \phi_{2}$ $:[0,1] \times \mathbb{R}^{2} \longrightarrow \mathbb{R}$ is continuous functions along with $p, q$ $\in L[0,1]$ which satisfy some certain linear growth conditions. By setting certain particular conditions, they derived their desired existence results using some techniques in Tdegree theory. The authors also investigated the HyersUlam stability for the proposed problem.

In [18], Khan et al. studied the existence of solutions and their uniqueness for the proposed coupled fractional structure of a FBVP having the nonlinear operator of $p$-Laplacian type and integral boundary conditions given by

$$
\begin{cases}{ }^{C} D_{0^{+}}^{r_{1}^{*}} \varphi_{p}\left({ }^{C} D_{0^{+}}^{r_{1}} v_{1}(t)\right)=\mathrm{e}_{1}\left(t, v_{1}, v_{2}(t)\right), & 0 \leq t \leq 1, \\ { }^{C} D_{0^{2}}^{r_{2}^{*}} \varphi_{p}\left({ }^{C} D_{0^{+}}^{r_{2}} v_{1}(t)\right)=\mathrm{e}_{2}\left(t, v_{1}, v_{2}(t)\right), & 0 \leq t \leq 1, \\ \varphi_{p}\left({ }^{C} D_{0^{+}}^{r_{1}} v_{1}\right)(0)=v_{1}^{\prime}(0)=0, & v_{1}(0)=c_{1} \frac{1}{\Gamma(\alpha-1)} \int_{0}^{K}(K-s)^{\alpha-2} v_{1}(s) d s, \\ \varphi_{p}\left({ }^{C} D_{0^{+}}^{r_{2}} v_{2}\right)(0)=v_{2}^{\prime}(0)=0, & v_{2}(0)=c_{2} \frac{1}{\Gamma(\beta-1)} \int_{0}^{K}(\eta-s)^{\beta-2} v_{2}(s) d s,\end{cases}
$$

where ${ }^{C} D_{0^{+}}^{r_{j}^{*}}(\cdot)$ and ${ }^{C} D_{0^{+}}^{r_{j}}(\cdot)$ for $j=1,2$ denote the Caputo derivative of orders $0<r_{j}^{*}<1$ and $1<r_{j}<2$. Additionally, $\varrho_{1}, \varrho_{2}:[0,1] \times \mathbb{R}^{2} \longrightarrow \mathbb{R}$ is continuous, and $\alpha, \beta \geq 1,-1 \leq c_{1}$ ,$c_{2} \leq 0$ and $\varphi_{p}(\theta)=|\theta|^{p-2} \theta$ stand for the $p$-Laplacian operator such that $1 / q+1 / p=1$. The authors established their desired theorems using the techniques attributed to LeraySchauder and Banach. Further, the Hyers-Ulam stability was investigated.

In [19] and by means of T-degree theory, Shah and Hussain established sufficient conditions for investigation of the existence of solutions and their stability on the following nonlinear FBVP

$$
\left\{\begin{array}{l}
{ }^{C} D_{0^{+}}^{r}=\Theta(t, \mu(t)), \quad 2<r<4, \\
\mu(0)=\zeta(t), \quad \mu^{\prime}(0)=\mu^{\prime \prime}(0)=0, \quad \mu(1)=v \mu(\eta),
\end{array}\right.
$$

where ${ }^{C} D_{0^{+}}^{r}(\cdot)$ represents the Caputo derivative of order $r$. Further, $\Theta:[0,1] \times \mathbb{R} \longrightarrow \mathbb{R}$ and $\zeta:[0,1] \longrightarrow \mathbb{R}$ are regarded to be continuous and $\mu, \eta \in(0,1)$.

By considering the existing literatures, we see that all differential equations having a $p$-Laplacian operator with threepoint integral boundary conditions are not well explored by T-degree theory, and even the boundary conditions of integral type cover a wide range of applications which have direct contributions in the theory of fluid mechanics, optimization, and viscoelasticity.

Inspired and motivated by the above fractional systems, we focus on the existence of solutions and establish four classes of Hyres-Ulam stability of a generalized FBVP having $p$-Laplacian operator with 3-point integral boundary conditions given by

$$
\begin{cases}{ }^{C} D^{\beta, \delta}\left(\varphi_{p}\left({ }^{C} D^{\chi, \rho} x(t)\right)\right)=\hbar_{1}(t, x(t)) & \left(t \in\left[t_{0}, K\right], t_{0} \geq 0\right), \\ x\left(t_{0}\right)+\mu_{1} x(K)=\sigma_{1} \int_{t_{0}}^{K} \hbar_{2}(s) d s, & \left(\mu_{1} \neq-1\right), \\ x^{\prime}\left(t_{0}\right)+\mu_{2} x^{\prime}(K)=\sigma_{2} \int_{t_{0}}^{K} \hbar_{3}(s) d s, & \left(\mu_{2} \neq-\left(\frac{t_{0}}{K}\right)^{\rho-1}\right), \\ { }^{C} D^{\chi, \rho} x\left(t_{0}\right)=0,{ }^{C} D^{\chi, \rho} x(K)=v^{C} D^{\chi, \rho} x(\eta), & \left(\eta \in\left(t_{0}, K\right)\right),\end{cases}
$$

so that ${ }^{C} D^{\beta, \delta}$ and ${ }^{C} D^{\chi, \rho}$ are generalized derivatives in the sense of Caputo, of order $\beta, \chi \in(1,2)$ and $\delta, \rho>0$. Along 
with these, $\mu_{1}, \mu_{2}, v, \sigma_{1}, \sigma_{2} \in \mathbb{R}$ with $v \neq\left(K^{\delta}-t_{0}^{\delta} / \eta^{\delta}-t_{0}^{\delta}\right)^{q-1}$ and also $\hbar_{2}, \hbar_{3}:\left[t_{0}, K\right] \longrightarrow \mathbb{R}$ and $\hbar_{1}:\left[t_{0}, K\right] \times \mathbb{R} \longrightarrow \mathbb{R}$ are assumed to be continuous. We emphasize that the proposed FBVP (4) has a novel structure and is designed for the first time in the context of a generalized fractional settings along with the $p$-Laplacian operator.

\section{Auxiliary Preliminaries}

The main purpose of this section is to collect some important definitions, primitive lemmas, and theoretical results of generalized fractional integrals and derivatives which are applicable in this paper.

By $\mathscr{C}\left(\left[t_{0}, K\right]\right)$, we mean the category of all continuous real functions defined on $\left[t_{0}, K\right]$ which is simply proved that it is a Banach space along with $\|x\|=\sup _{t \in\left[t_{0}, K\right]}|x(t)|$. Moreover, $A C^{l}\left(\left[t_{0}, K\right], \mathbb{R}\right)=\left\{x:\left[t_{0}, K\right] \longrightarrow \mathbb{R}: x^{(l-1)}(t) \in A C\left(\left[t_{0}\right.\right.\right.$, $K], \mathbb{R})\}$ stands for the space of absolutely continuous functions on $\left[t_{0}, K\right]$ having real values up to $(l-1)$-derivative. Thus, in this regard, we define

$$
\begin{aligned}
& A C_{\delta}^{l}\left(\left[t_{0}, K\right], \mathbb{R}\right) \\
& \quad=\left\{x:\left[t_{0}, K\right] \longrightarrow \mathbb{R}:\left(\delta^{l-1} x\right)(t) \in A C\left(\left[t_{0}, K\right], \mathbb{R}\right), \delta=\frac{1}{t^{\rho-1}} \frac{d}{d t}\right\},
\end{aligned}
$$

as a category of functions having absolutely continuous $\delta^{l-1}$ -derivatives, and a norm is defined by

$$
\|x\|_{C_{\delta}^{l}}=\sum_{k=0}^{l-1}\left\|\left(\delta^{k} x\right)(t)\right\|_{C}
$$

so that $\delta^{k}=\delta \delta \cdots \delta^{k \text {-times }}$.

Definition 1. (see [20]). Let $0<a, b<+\infty, q>0$ and $x \in \mathscr{X}_{c}^{p}$ $(a, b)$, where $\mathscr{X}_{c}^{p}(a, b)$ is the space of all Lebesgue measurable complex functions. The integral operator given by

$$
{ }^{\rho} I_{a^{+}}^{q} x(t)=\frac{\rho^{1-q}}{\Gamma(q)} \int_{a^{+}}^{t} s^{\rho-1}\left(t^{\rho}-s^{\rho}\right)^{q-1} x(s) d s, \rho>0, t>a^{+}
$$

is named as the generalized Riemann-Liouville integral such that the R.H.S. integral is finite-valued.

Definition 2. (see [20]). The generalized derivative in sense of Caputo for a given function $f \in A C_{\delta}^{l}[a, b]$ of order $l-1<q$ $<l$ with $l=[q]+1$ is defined by

$$
\begin{aligned}
{ }^{C} D_{a^{+}}^{q, \rho} x(t)= & { }^{\rho} I_{a^{+}}^{l-q}\left(\delta^{l} f\right)(t)=\frac{\rho^{q-l+1}}{\Gamma(l-q)} \int_{a^{+}}^{t} s^{\rho-1}\left(t^{\rho}-s^{\rho}\right)^{l-q-1} \\
& \cdot\left(\delta^{l} f\right)(s) d s,\left(\rho>0, t>a^{+}\right)
\end{aligned}
$$

In particular, if $q=l \in \mathbb{N}$,

$$
{ }^{C} D_{a^{+}}^{q, \rho} x(t)=\left(\delta^{l} f\right)(s)
$$

Lemma 3. (see [20]). Let $f \in A C_{\delta}^{l}[a, b]$. Then, for every $l-1$ $<q<l$,

$$
{ }^{\rho} I_{a^{+}}^{q C} D_{a^{+}}^{q, \rho} x(t)=x(t)-\sum_{k=0}^{l-1} \frac{\left(\delta^{k} f\right)(a)}{\rho^{k} k !}\left(t^{\rho}-a^{\rho}\right)^{k} .
$$

Moreover, for $0<q<1$, (10) becomes

$$
{ }^{\rho} I_{a^{+}}^{q C} D_{a^{+}}^{q, \rho} x(t)=x(t)-x(a)
$$

Now, we will present a definition of Kuratowski's measure of noncompactness $\xi(\cdot)$ which is constructed by

$\xi(\mathscr{D})=\inf \left\{\varepsilon>0: \mathscr{D}=\bigcup_{i=1}^{n} \mathscr{D}_{i}\right.$ and $\operatorname{Diam}\left(\mathscr{D}_{i}\right) \leq \varepsilon$ for $\left.i=1, \cdots, n\right\}$,

where $\operatorname{Diam}\left(\mathscr{D}_{i}\right)=\sup \left\{|x-y|: x, y \in \mathscr{D}_{i}\right\}$ and $\mathscr{D}$ are a bounded subset of the Banach space $\mathscr{C}\left(\left[t_{0}, K\right]\right)$. It is clear that $0 \leq \xi(\mathscr{D}) \leq \operatorname{Diam}(\mathscr{D})<+\infty[21]$.

Definition 4. (see [21]). Let $\mathscr{G}: \mathscr{V} \longrightarrow \mathscr{W}$ be bounded and continuous with $\mathscr{V} \subset \mathscr{W}$. Then, $\mathscr{G}$ will be $\xi$-Lipschitz if $\exists \varsigma$ $\geq 0$ so that

$$
\xi(\mathscr{G}(A))<\varsigma \xi(A), \forall \text { bounded } A \subset \mathscr{V}
$$

As well as $\mathscr{G}$ is named as strict $\xi$-contraction when $\varsigma<1$ holds.

Definition 5. (see [21]). A function $\mathscr{G}$ is $\xi$-condensing if

$$
\xi(\mathscr{G}(A))<\xi(A), \forall A \subset \mathscr{V} \text { bounded, with } \xi(A)>0 .
$$

So, $\xi(\mathscr{G}(A)) \geq \xi(A)$ gives $\xi(A)=0$. Also, $\mathscr{G}: \mathscr{V} \longrightarrow \mathscr{W}$ is Lipschitz for $\varsigma>0$ such that

$$
\left\|\mathscr{G}(\mu)-\mathscr{G}\left(\mu^{\prime}\right)\right\| \leq \varsigma\left\|\mu-\mu^{\prime}\right\| \text { for all } \mu, \mu^{\prime} \in \mathscr{V} \text {. }
$$

If $\varsigma<1$, in this case $\mathscr{G}$ is called a strict contraction.

Proposition 6. (see [21]). $\mathscr{G}$ is $\xi$-Lipschitz with constant $\varsigma=0$ iff $\mathscr{G}: \mathscr{V} \longrightarrow \mathscr{W}$ is compact.

Proposition 7. (see [21]). A function $\mathscr{G}$ is $\xi$-Lipschitz with constant $\varsigma$ if and only if $\mathscr{G}: \mathscr{V} \longrightarrow \mathscr{W}$ is Lipschitz with Lipschitz constant $\varsigma$.

Theorem 8. (see [22]). Let $\mathscr{G}: \mathscr{C}\left[t_{0}, K\right] \longrightarrow \mathscr{C}\left[t_{0}, K\right]$ be a $\xi$ -condensing and 
$\mathbb{M}=\left\{u \in \mathscr{C}\left[t_{0}, K\right]: \theta \in[0,1]\right.$ exists so that $\left.u=\theta \mathscr{G}(u)\right\}$

If $\mathbb{M}$ is a bounded subset contained in $\mathscr{C}\left[t_{0}, K\right]$, i.e., a constant $r>0$ exists with $\mathbb{M} \subset K_{r}(0)$, then $\operatorname{deg}\left(I-\theta \mathscr{G}, K_{r}(0), 0\right)$ $=1$ for all $\theta \in[0,1]$. Therefore, $\mathscr{G}$ has a fixed point, and the set $\mathbb{F} \mathbb{X}(\mathscr{G})$ belongs to $K_{r}(0)$.

Lemma 9. (see [13]). Consider $\varphi_{p}$ as an operator in the $p$ Laplacian settings. then

(p1) For $1<p \leq 2$, if $Y_{1}, Y_{2}>0$ and $\left|Y_{1}\right|,\left|Y_{2}\right| \geq \lambda>0$,

$$
\left|\varphi_{p}\left(Y_{1}\right)-\varphi_{p}\left(Y_{2}\right)\right| \leq(p-1) \lambda^{p-2}\left|Y_{1}-Y_{2}\right|
$$

(p2) For $p>2$, if $\left|Y_{1}\right|,\left|Y_{2}\right| \leq \lambda^{*}$, then

$$
\left|\varphi_{p}\left(Y_{1}\right)-\varphi_{p}\left(Y_{2}\right)\right| \leq(p-1) \lambda^{* p-2}\left|Y_{1}-Y_{2}\right|
$$

\section{Main Analytical Results}

This important section is divided into some subsections. In each part, we shall study desired theorems about different specifications of solutions to the proposed $p$-Laplacian FBVP (4).

3.1. Existence-Uniqueness Results. We here present the first result which yields the solution of the proposed $p$-Laplacian FBVP (4) in the equivalent format of integral equations.
Theorem 10. Let $\hbar_{1}, \hbar_{2}, \hbar_{3} \in \mathscr{C}\left[t_{0}, K\right], K>t_{0} \geq 0, \chi, \beta \in(1,2)$, and $\delta, \rho, v>0$. The p-Laplacian FBVP with given integral composite conditions

$$
\left\{\begin{array}{l}
{ }^{C} D^{\beta, \delta}\left(\varphi_{p}\left({ }^{C} D^{\chi, \rho} x(t)\right)\right)=\hbar_{1}(t), \\
x\left(t_{0}\right)+\mu_{1} x(K)=\sigma_{1} \int_{t_{0}}^{K} g(s) d s, \\
x^{\prime}\left(t_{0}\right)+\mu_{2} x^{\prime}(K)=\sigma_{2} \int_{t_{0}}^{K} \hbar_{3}(s) d s, \\
{ }^{C} D^{\chi, \rho} x\left(t_{0}\right)=0,{ }^{C} D^{\chi, \rho} x(K)=v^{C} D^{\chi, \rho} x(\eta),
\end{array}\right.
$$

has a solution given by

$$
\begin{aligned}
x(t)= & \int_{t_{0}}^{K}\left(H_{1}(t, \omega) \varphi_{q}\left(\int_{t_{0}}^{K} H_{2}(\omega, s) \hbar_{1}(s) d s\right)+\frac{\sigma_{1} \hbar_{2}(\omega)}{1+\mu_{1}}\right. \\
& \left.+\frac{\sigma_{2} \hbar_{3}(\omega)}{t_{0}^{\rho-1}+\mu_{2} K^{\rho-1}}\left(\frac{t^{\rho}-t_{0}^{\rho}}{\rho}-\frac{\mu_{1}}{1+\mu_{1}} \frac{K^{\rho}-t_{0}^{\rho}}{\rho}\right)\right) d \omega,
\end{aligned}
$$

such that $H_{1}(t, \omega)$ and $H_{2}(t, s)$ stand for Green's functions defined as follows:

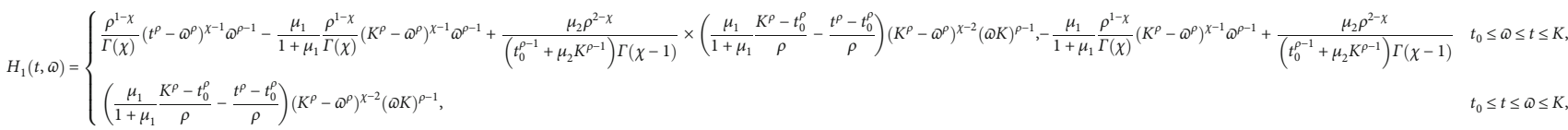

$$
\begin{aligned}
& H_{2}(t, s)=\left\{\begin{array}{lr}
\frac{\delta^{1-\beta}}{\Gamma(\beta)}\left(t^{\delta}-s^{\delta}\right)^{\beta-1} s^{\delta-1}-\frac{\delta^{1-\beta}\left(t^{\delta}-t_{0}^{\delta}\right)}{\left(\left(K^{\delta}-t_{0}^{\delta}\right)-v^{1 / q-1}\left(\eta^{\delta}-t_{0}^{\delta}\right)\right) \Gamma(\beta)}\left(K^{\delta}-s^{\delta}\right)^{\beta-1} s^{\delta-1}+\frac{\delta^{1-\beta} v^{1 / q-1}\left(t^{\delta}-t_{0}^{\delta}\right)}{\left(\left(K^{\delta}-t_{0}^{\delta}\right)-v^{1 / q-1}\left(\eta^{\delta}-t_{0}^{\delta}\right)\right) \Gamma(\beta)}\left(\eta^{\delta}-s^{\delta}\right)^{\beta-1} s^{\delta-1}, & t_{0} \leq s \leq \eta \leq t, \leq K, \\
\frac{\delta^{1-\beta}}{\Gamma(\beta)}\left(t^{\delta}-s^{\delta}\right)^{\beta-1} s^{\delta-1}-\frac{\delta^{1-\beta}\left(t^{\delta}-t_{0}^{\delta}\right)\left(K^{\delta}-s^{\delta}\right)^{\beta-1} s^{\delta-1}}{\left(\left(K^{\delta}-t_{0}^{\delta}\right)-v^{1 / q-1}\left(\eta^{\delta}-t_{0}^{\delta}\right)\right) \Gamma(\beta)} & t_{0} \leq \eta \leq s \leq t, \leq K, \\
-\frac{\delta^{1-\beta}\left(t^{\delta}-t_{0}^{\delta}\right)}{\left(\left(K^{\delta}-t_{0}^{\delta}\right)-v^{1 / q-1}\left(\eta^{\delta}-t_{0}^{\delta}\right)\right) \Gamma(\beta)}\left(K^{\delta}-s^{\delta}\right)^{\beta-1} s^{\delta-1}, & t_{0} \leq \eta \leq t \leq s . \leq K .
\end{array}\right.
\end{aligned}
$$

Proof. By utilizing the integral operator ${ }^{\delta} I^{\beta}$ on (19), the following relation is produced

$$
\varphi_{p}\left({ }^{C} D^{\chi, \rho} x(t)\right)={ }^{\delta} I^{\beta} \hbar_{1}(t)-a_{1}-a_{2}\left(\frac{t^{\delta}-t_{0}^{\delta}}{\delta}\right), a_{1}, a_{2} \in \mathbb{R} .
$$
have

By taking the inverse $\varphi_{q}$ of $\varphi_{p}$ on above equation, we

$$
\begin{aligned}
{ }^{C} D^{\chi, \rho} x(t)= & \varphi_{q}\left(\frac{\delta^{1-\beta}}{\Gamma(\beta)} \int_{t_{0}}^{t}\left(t^{\delta}-s^{\delta}\right)^{\beta-1} s^{\delta-1} \hbar_{1}(s) d s\right. \\
& \left.-a_{1}-a_{2}\left(\frac{t^{\delta}-t_{0}^{\delta}}{\delta}\right)\right) .
\end{aligned}
$$

Using the boundary conditions ${ }^{C} D^{\chi, \rho} x\left(t_{0}\right)=0$ and ${ }^{C} D^{\chi, \rho} x(K)=v^{C} D^{\chi, \rho} x(\eta)$, we have $a_{1}=0$ and 


$$
\begin{aligned}
& \varphi_{q}\left(\frac{\delta^{1-\beta}}{\Gamma(\beta)} \int_{t_{0}}^{K}\left(K^{\delta}-s^{\delta}\right)^{\beta-1} s^{\delta-1} \hbar_{1}(s) d s-a_{2}\left(\frac{K^{\delta}-t_{0}^{\delta}}{\delta}\right)\right) \\
& =v \varphi_{q}\left(\frac{\delta^{1-\beta}}{\Gamma(\beta)} \int_{t_{0}}^{\eta}\left(\eta^{\delta}-s^{\delta}\right)^{\beta-1} s^{\delta-1} \hbar_{1}(s) d s-a_{2}\left(\frac{\eta^{\delta}-t_{0}^{\delta}}{\delta}\right)\right) \\
& =\varphi_{q}\left(\frac{v^{1 / q-1} \delta^{1-\beta}}{\Gamma(\beta)} \int_{t_{0}}^{\eta}\left(\eta^{\delta}-s^{\delta}\right)^{\beta-1} s^{\delta-1} \hbar_{1}(s) d s-a_{2} v^{\frac{1}{q-1}}\left(\frac{\eta^{\delta}-t_{0}^{\delta}}{\delta}\right)\right)
\end{aligned}
$$

which is obtained by using the property of $p$-Laplacian operator. Therefore,

$$
\begin{aligned}
& a_{2}\left(\left(\frac{K^{\delta}-t_{0}^{\delta}}{\delta}\right)-v^{\frac{1}{q-1}}\left(\frac{\eta^{\delta}-t_{0}^{\delta}}{\delta}\right)\right) \\
& =\frac{\delta^{1-\beta}}{\Gamma(\beta)} \int_{t_{0}}^{K}\left(K^{\delta}-s^{\delta}\right)^{\beta-1} s^{\delta-1} \hbar_{1}(s) d s-\frac{v^{1 / q-1} \delta^{1-\beta}}{\Gamma(\beta)} \int_{t_{0}}^{\eta} \\
& \cdot\left(\eta^{\delta}-s^{\delta}\right)^{\beta-1} s^{\delta-1} \hbar_{1}(s) d s,
\end{aligned}
$$

which implies that

$$
\begin{aligned}
a_{2}= & \frac{\delta^{2-\beta}}{\left(\left(K^{\delta}-t_{0}^{\delta}\right)-v^{1 / q-1}\left(\eta^{\delta}-t_{0}^{\delta}\right)\right) \Gamma(\beta)} \int_{t_{0}}^{K} \\
& \cdot\left(K^{\delta}-s^{\delta}\right)^{\beta-1} s^{\delta-1} \hbar_{1}(s) d s \\
& -\frac{v^{1 / q-1} \delta^{2-\beta}}{\left(\left(K^{\delta}-t_{0}^{\delta}\right)-v^{1 / q-1}\left(\eta^{\delta}-t_{0}^{\delta}\right)\right) \Gamma(\beta)} \int_{t_{0}}^{\eta} \\
& \cdot\left(\eta^{\delta}-s^{\delta}\right)^{\beta-1} s^{\delta-1} \hbar_{1}(s) d s .
\end{aligned}
$$

According to (24) and the definition of $\mathrm{H}_{2}(t, s)$, we get

$$
{ }^{C} D^{\chi, \rho} x(t)=\varphi_{q}\left(\int_{t_{0}}^{K} H_{2}(t, s) \hbar_{1}(s) d s\right)
$$

Applying the integral operator ${ }^{\rho} I^{\chi}$, we shall write

$$
x(t)={ }^{\rho} I^{\chi} \varphi_{q}\left(\int_{t_{0}}^{K} H_{2}(t, s) \hbar_{1}(s) d s\right)-b_{1}-b_{2} \frac{t^{\rho}-t_{0}^{\rho}}{\rho} .
$$

By virtue of the boundary condition $x\left(t_{0}\right)+\mu_{1} x(K)=$ $\sigma_{1} \int_{t_{0}}^{K} \hbar_{2}(s) d s$, we have

$$
-b_{1}+\mu_{1}\left(I^{\chi, \rho} \varphi_{q}\left(\int_{t_{0}}^{K} H_{2}(t, s) \hbar_{1}(s) d s\right)-b_{1}-b_{2} \frac{K^{\rho}-t_{0}^{\rho}}{\rho}\right)=\sigma_{1} \int_{t_{0}}^{K} \hbar_{2}(s) d s
$$

which implies

$$
\begin{aligned}
b_{1}= & \frac{\mu_{1}}{1+\mu_{1}} \frac{\rho^{1-\chi}}{\Gamma(\chi)} \int_{t_{0}}^{K}\left(K^{\rho}-\omega^{\rho}\right)^{\chi-1} \omega^{\rho-1} \varphi_{q}\left(\int_{t_{0}}^{K} H_{2}(\varrho, s) \hbar_{1}(s) d s\right) d \omega \\
& -\frac{\mu_{1}}{1+\mu_{1}} \frac{K^{\rho}-t_{0}^{\rho}}{\rho} b_{2}-\frac{\sigma_{1}}{1+\mu_{1}} \int_{t_{0}}^{K} \hbar_{2}(s) d s .
\end{aligned}
$$

Therefore, we obtain

$$
\begin{aligned}
x^{\prime}(t)= & \frac{\rho^{2-\chi}}{\Gamma(\chi-1)} \int_{t_{0}}^{t}\left(t^{\rho}-\omega^{\rho}\right)^{\chi-2}(t \omega)^{\rho-1} \varphi_{q} \\
& \cdot\left(\int_{t_{0}}^{K} H_{2}(\omega, s) \hbar(s) d s\right) d \omega-b_{2} t^{\rho-1} .
\end{aligned}
$$

Using the boundary condition $x^{\prime}\left(t_{0}\right)+\mu_{2} x^{\prime}(K)=\sigma_{2} \int_{t_{0}}^{K}$ $\hbar_{3}(s) d s$,

$$
\begin{gathered}
-b_{2} t_{0}^{\rho-1}+\mu_{2} \frac{\rho^{2-\chi}}{\Gamma(\chi-1)} \int_{t_{0}}^{K}\left(K^{\rho}-\omega^{\rho}\right)^{\chi-2}(\bowtie K)^{\rho-1} \varphi_{q} \\
\cdot\left(\int_{t_{0}}^{K} H_{2}(\omega, s) \hbar_{1}(s) d s\right) d \omega-\mu_{2} b_{2} K^{\rho-1}=\sigma_{2} \int_{t_{0}}^{K} \hbar_{3}(s) d s .
\end{gathered}
$$

Then,

$$
\begin{aligned}
b_{2}= & \frac{\mu_{2}}{t_{0}^{\rho-1}+\mu_{2} K^{\rho-1}} \frac{\rho^{2-\chi}}{\Gamma(\chi-1)} \int_{t_{0}}^{K}\left(K^{\rho}-\omega^{\rho}\right)^{\chi-2}(\omega K)^{\rho-1} \varphi_{q} \\
& \cdot\left(\int_{t_{0}}^{K} H_{2}(\omega, s) \hbar_{1}(s) d s\right) d \omega-\frac{\sigma_{2}}{t_{0}^{\rho-1}+\mu_{2} K^{\rho-1}} \int_{t_{0}}^{K} \hbar_{3}(s) d s .
\end{aligned}
$$

Therefore,

$$
\begin{aligned}
x(t)= & \frac{\rho^{1-\chi}}{\Gamma(\chi)} \int_{t_{0}}^{t}\left(t^{\rho}-\omega^{\rho}\right)^{\chi-1} \omega^{\rho-1} \varphi_{q}\left(\int_{t_{0}}^{K} H_{2}(\omega, s) \hbar_{1}(s) d s\right) d \omega \\
& -\frac{\mu_{1}}{1+\mu_{1}} \frac{\rho^{1-\chi}}{\Gamma(\chi)} \int_{t_{0}}^{K}\left(K^{\rho}-\omega^{\rho}\right)^{\chi-1} \omega^{\rho-1} \varphi_{q} \\
& \cdot\left(\int_{t_{0}}^{K} H_{2}(\omega, s) \hbar_{1}(s) d s\right) d \omega+\frac{\mu_{2} \rho^{2-\chi}}{\left(t_{0}^{\rho-1}+\mu_{2} K^{\rho-1}\right) \Gamma(\chi-1)} \\
& \cdot\left(\frac{\mu_{1}}{1+\mu_{1}} \frac{K^{\rho}-t_{0}^{\rho}}{\rho}-\frac{t^{\rho}-t_{0}^{\rho}}{\rho}\right) \times \int_{t_{0}}^{K}\left(K^{\rho}-\omega^{\rho}\right)^{\chi-2}(\omega K)^{\rho-1} \varphi_{q} \\
& \cdot\left(\int_{t_{0}}^{K} H_{2}(\omega, s) \hbar_{1}(s) d s\right) d \omega+\frac{\sigma_{1}}{1+\mu_{1}} \int_{t_{0}}^{K} \hbar_{2}(\omega) d \omega \\
& +\frac{\sigma_{2}}{t_{0}^{\rho-1}+\mu_{2} K^{\rho-1}}\left(\frac{t^{\rho}-t_{0}^{\rho}}{\rho}-\frac{\mu_{1}}{1+\mu_{1}} \frac{K^{\rho}-t_{0}^{\rho}}{\rho}\right) \int_{t_{0}}^{K} \hbar_{3}(\omega) d \omega \\
= & \int_{t_{0}}^{K}\left(H_{1}(t, \omega) \varphi_{q}\left(\int_{t_{0}}^{K} H_{2}(\omega, s) \hbar_{1}(s) d s\right)+\frac{\sigma_{1} \hbar_{2}(\omega)}{1+\mu_{1}}\right. \\
& \left.+\frac{\sigma_{2} h(\omega)}{t_{0}^{\rho-1}+\mu_{2} K^{\rho-1}}\left(\frac{t^{\rho}-t_{0}^{\rho}}{\rho}-\frac{\mu_{1}}{1+\mu_{1}} \frac{K^{\rho}-t_{0}^{\rho}}{\rho}\right)\right) d \omega,
\end{aligned}
$$

where $H_{1}(t, \varpi)$ is defined in (18). This ends the proof. 
In this part, we intend to state and prove our required existence-uniqueness theorems. To achieve such an intention and in view of Theorem 10, the solution of the suggested $p$-Laplacian FBVP (4) is equivalent to a fixed point $x(t)$ of the self-map $\Xi: \mathscr{C}\left[t_{0}, K\right] \longrightarrow \mathscr{C}\left[t_{0}, K\right]$ which is formulated as

$$
\begin{aligned}
(\Xi x)(t)= & \int_{t_{0}}^{K}\left(H_{1}(t, \varrho) \varphi_{q}\left(\int_{t_{0}}^{K} H_{2}(\varrho, s) \hbar_{1}(s, x(s)) d s\right)\right. \\
& \left.+\frac{\sigma_{1} \hbar_{2}(\Phi)}{1+\mu_{1}}+\frac{\sigma_{2} \hbar_{3}(\Phi)}{t_{0}^{\rho-1}+\mu_{2} K^{\rho-1}}\left(\frac{t^{\rho}-t_{0}^{\rho}}{\rho}-\frac{\mu_{1}}{1+\mu_{1}} \frac{K^{\rho}-t_{0}^{\rho}}{\rho}\right)\right) d \omega,
\end{aligned}
$$

where $H_{1}(t, \omega)$ and $H_{2}(\omega, s)$ are represented by (21) and (22), respectively. In the sequel, we utilize the following notations:

$$
\Delta_{1}=\left(\frac{K^{\rho}-t_{0}{ }^{\rho}}{\rho}\right)^{\chi}\left(\left|\frac{\mu_{1}}{1+\mu_{1}}\right|+1\right)\left(\frac{1}{\Gamma(\chi+1)}+\frac{\left|\mu_{2}\right| K^{\rho-1}}{\Gamma(\chi)\left|\left(t_{0}^{\rho-1}+\mu_{2} K^{\rho-1}\right)\right|}\right)
$$$$
\begin{aligned}
\Delta_{2}= & \left(\frac{K^{\delta}-t_{0}^{\delta}}{\delta}\right)^{\beta} \frac{1}{\Gamma(\beta+1)}+\frac{\left(K^{\delta}-t_{0}^{\delta}\right)}{\left|\left(\left(K^{\delta}-t_{0}^{\delta}\right)-v^{1 / q-1}\left(\eta^{\delta}-t_{0}^{\delta}\right)\right)\right|} \\
& \cdot\left(\left(\frac{K^{\delta}-t_{0}^{\delta}}{\delta}\right)^{\beta} \frac{1}{\Gamma(\beta+1)}+\left(\frac{\eta^{\delta}-t_{0}^{\delta}}{\delta}\right)^{\beta} \frac{v^{1 / q-1}}{\Gamma(\beta+1)}\right),
\end{aligned}
$$$$
\Delta_{3}=\left|\frac{\sigma_{1}}{1+\mu_{1}}\right| \hbar_{2}^{*}+\frac{\left|\sigma_{2}\right| \hbar_{3}^{*}}{\left|t_{0}^{\rho-1}+\mu_{2} K^{\rho-1}\right|}\left(\frac{K^{\rho}-t_{0}^{\rho}}{\rho}\right)\left(\left|\frac{\mu_{1}}{1+\mu_{1}}\right|+1\right),
$$

and $\omega_{1}^{*}=\sup _{t \in\left[t_{0}, K\right]}\left\{\omega_{1}(t)\right\}, \omega_{2}^{*}=\sup _{t \in\left[t_{0}, K\right]}\left\{\omega_{2}(t)\right\}, \Omega_{1}=\omega_{1}^{*}$ $\Delta_{1} \Delta_{2}^{q-1}+\Delta_{3}, \Omega_{2}=\omega_{2}^{*} \Delta_{1} \Delta_{2}^{q-1}, y_{2}^{*}=\sup _{t \in\left[t_{0}, K\right]}\left|\hbar_{2}(t)\right|$, and $\hbar_{3}^{*}$ $=\sup _{t \in\left[t_{0}, K\right]}\left|\hbar_{3}(t)\right|$.

Theorem 11. Let (HP1): the functions $\omega_{1}, \omega_{2} \in \mathscr{C}\left[t_{0}, K\right]$ exist so that $\left|\hbar_{1}(t, x)\right| \leq \varphi_{p}\left(\omega_{1}(t)+\omega_{2}(t)|x(t)|\right)$ for any $x \in \mathscr{C}\left[t_{0}\right.$, $K]$ and $t \in\left[t_{0}, K\right]$.

Then, $\Xi: \mathscr{C}\left[t_{0}, K\right] \longrightarrow \mathscr{C}\left[t_{0}, K\right]$ is continuous, and also the growth condition $\|\Xi x\| \leq \Omega_{1}+\Omega_{2}\|x\|$ holds.

Proof. Define a set $\mathfrak{B}_{\varepsilon}=\left\{x \in \mathscr{C}\left[t_{0}, K\right]:\|x\| \leq \varepsilon\right\}$ having the boundedness property. In order to prove the continuity of $\Xi$, we consider $x_{n}$ as a sequence converging to $x$ in $\mathfrak{B}_{\varepsilon}$. Then, Lemma 9 yields

$$
\begin{aligned}
& \left|\left(\Xi x_{n}\right)(t)-(\Xi x)(t)\right| \\
& =\mid \int_{t_{0}}^{K}\left(H_{1}(t, \varpi) \varphi_{q}\left(\int_{t_{0}}^{K} H_{2}(\omega, s) \hbar_{1}\left(s, x_{n}(s)\right) d s\right) d \omega\right. \\
& -\int_{t_{0}}^{K}\left(H_{1}(t, \varpi) \varphi_{q}\left(\int_{t_{0}}^{K} H_{2}(\varrho, s) \hbar_{1}(s, x(s)) d s\right) d \Phi \mid\right. \\
& \leq \int_{t_{0}}^{K}\left|H_{1}(t, \emptyset)\right| \mid \varphi_{q}\left(\int_{t_{0}}^{K} H_{2}(\varrho, s) \hbar_{1}\left(s, x_{n}(s)\right) d s\right) \\
& -\varphi_{q}\left(\int_{t_{0}}^{K} H_{2}(\varrho, s) \hbar_{1}(s, x(s)) d s\right) \mid d \omega \\
& \leq(q-1) \lambda^{q-2} \int_{t_{0}}^{K}\left|H_{1}(t, \omega)\right| \\
& \cdot\left(\int_{t_{0}}^{K}\left|H_{2}(\varrho, s)\right|\left|\hbar_{1}\left(s, x_{n}(s)\right)-\hbar_{1}(s, x(s))\right| d s\right) d \omega .
\end{aligned}
$$

According to Lebesgue's dominated convergence theorem and the continuity of the function $\hbar_{1}$, we get $\| \Xi x_{n}-\Xi$ $x \| \longrightarrow 0$ when $n \longrightarrow \infty$. Hence, $\Xi$ is continuous.

Now, about the growth condition, by (HP1), we obtain

$$
\begin{aligned}
& |(\Xi x)(t)| \leq \int_{t_{0}}^{K}\left(\left|H_{1}(t, \varpi)\right| \varphi_{q}\left(\int_{t_{0}}^{K}\left|H_{2}(\Phi, s)\right|\left|\hbar_{1}(s, x(s))\right| d s\right)\right. \\
& \left.+\left|\frac{\sigma_{1} \hbar_{2}(\Phi)}{1+\mu_{1}}\right|+\left|\frac{\sigma_{2} \hbar_{3}(\Phi)}{t_{0}^{\rho-1}+\mu_{2} K^{\rho-1}}\left(\frac{t^{\rho}-t_{0}^{\rho}}{\rho}-\frac{\mu_{1}}{1+\mu_{1}} \frac{K^{\rho}-t_{0}^{\rho}}{\rho}\right)\right|\right) d \Phi \\
& \leq \int_{t_{0}}^{K}\left(\left|H_{1}(t, \varpi)\right| \varphi_{q}\left(\int_{t_{0}}^{K}\left|H_{2}(\varpi, s)\right| \varphi_{p}\left(\omega_{1}(s)+\omega_{2}(s)|x(s)|\right) d s\right)\right. \\
& \left.+\left|\frac{\sigma_{1} \hbar_{2}(\Phi)}{1+\mu_{1}}\right|+\left|\frac{\sigma_{2} \hbar_{3}(\Phi)}{t_{0}^{\rho-1}+\mu_{2} K^{\rho-1}}\left(\frac{t^{\rho}-t_{0}^{\rho}}{\rho}-\frac{\mu_{1}}{1+\mu_{1}} \frac{K^{\rho}-t_{0}^{\rho}}{\rho}\right)\right|\right) d \omega \\
& \leq \int_{t_{0}}^{K}\left(\left|H_{1}(t, \omega)\right| \Delta_{2}^{q-1}\left(\omega_{1}^{*}+\omega_{2}^{*}\|x\|\right)+\left|\frac{\sigma_{1} \hbar_{2}(\Phi)}{1+\mu_{1}}\right|\right. \\
& \left.+\left|\frac{\sigma_{2} \hbar_{3}(\Phi)}{t_{0}^{\rho-1}+\mu_{2} K^{\rho-1}}\left(\frac{t^{\rho}-t_{0}^{\rho}}{\rho}-\frac{\mu_{1}}{1+\mu_{1}} \frac{K^{\rho}-t_{0}^{\rho}}{\rho}\right)\right|\right) d \omega \\
& \leq \Delta_{1} \Delta_{2}^{q-1}\left(\omega_{1}^{*}+\omega_{2}^{*}\|x\|\right)+\Delta_{3} \leq \omega_{1}^{*} \Delta_{1} \Delta_{2}^{q-1}+\Delta_{3}+\omega_{2}^{*} \Delta_{1} \Delta_{2}^{q-1}\|x\| \\
& \leq \Omega_{1}+\Omega_{2}\|x\| \text {. }
\end{aligned}
$$

Thus, $\|\Xi x\| \leq \Omega_{1}+\Omega_{2}\|x\|$ and this complete the argument.

Theorem 12. Under hypothesis (HP1), the single-valued operator $\Xi: \mathscr{C}\left[t_{0}, K\right] \longrightarrow \mathscr{C}\left[t_{0}, K\right]$ is $\xi$-Lipschitz with the constant zero and is compact.

Proof. In view of Theorem 11, $\Xi$ is bounded. In the subsequent step, we show that $\Xi$ is an equicontinuous operator. Then, by the hypothesis (HP1), for any $x \in \mathfrak{B}_{\varepsilon}$ and $t_{1}, t_{2}$ $\in\left[t_{0}, K\right]$ subject to $t_{1}<t_{2}$, we have 


$$
\begin{aligned}
& \left|(\Xi x)\left(t_{2}\right)-(\Xi x)\left(t_{1}\right)\right| \leq \mid \int_{t_{0}}^{K}\left(H_{1}\left(t_{2}, \varpi\right) \varphi_{q}\left(\int_{t_{0}}^{K} H_{2}(\omega, s) \hbar_{1}(s, x(s)) d s\right)\right. \\
& \left.+\frac{\sigma_{2} \hbar_{3}(\omega)}{t_{0}^{\rho-1}+\mu_{2} K^{\rho-1}}\left(\frac{t_{2}^{\rho}-t_{0}^{\rho}}{\rho}\right)\right) d \omega-\int_{t_{0}}^{K} \\
& \cdot\left(H_{1}\left(t_{1}, \omega\right) \varphi_{q}\left(\int_{t_{0}}^{K} H_{2}(\varrho, s) \hbar_{1}(s, x(s)) d s\right)\right. \\
& \left.+\frac{\sigma_{2} \hbar_{3}(\varpi)}{t_{0}^{\rho-1}+\mu_{2} K^{\rho-1}}\left(\frac{t_{1}^{\rho}-t_{0}^{\rho}}{\rho}\right)\right) d \omega \mid \leq \int_{t_{0}}^{K}\left(\left|H_{1}\left(t_{2}, \varpi\right)-H_{1}\left(t_{1}, \varpi\right)\right| \varphi_{q}\right. \\
& \cdot\left(\int_{t_{0}}^{K}\left|H_{2}(\varrho, s)\right| \varphi_{p}\left(\omega_{1}(s)+\omega_{2}(s)|x(s)|\right) d s\right) \\
& \left.+\frac{\left|\sigma_{2} \hbar_{3}(\varpi)\right|}{\left|t_{0}^{\rho-1}+\mu_{2} K^{\rho-1}\right|}\left(\frac{t_{2}^{\rho}-t_{0}^{\rho}}{\rho}-\frac{t_{1}^{\rho}-t_{0}^{\rho}}{\rho}\right)\right) d \omega .
\end{aligned}
$$

Clearly, the R.H.S. of (40) goes to zero by taking $t_{2}$ $\longrightarrow t_{1}$, and so $\Xi\left(\mathfrak{B}_{\varepsilon}\right)$ is equicontinuous. Therefore, by virtue of the well-known Arzelá-Ascoli theorem, $\Xi\left(\mathfrak{B}_{\varepsilon}\right)$ is compact, and thus Proposition 6 gives a result stating this fact that $\Xi$ is $\xi$-Lipschitz with the constant zero.

Theorem 13. Under the following hypothesis, i.e.,

(HP2) A real constant $\ell$ exists so that for any $\mu_{1}, \mu_{2} \in \mathscr{C}$ $\left[t_{0}, K\right]$ and $t \in\left[t_{0}, K\right]$,

$$
\left|\hbar_{1}\left(t, \mu_{1}\right)-\hbar_{1}\left(t, \mu_{2}\right)\right| \leq \ell\left|\mu_{1}(t)-\mu_{2}(t)\right| .
$$

The generalized p-Laplacian FBVP (4) has a unique solution such that

$$
\ell(q-1) \lambda^{q-2} \Delta_{1} \Delta_{2}<1
$$

Proof. Consider $\Xi$ as defined in (36). Then by Lemma 9, we obtain

$$
\begin{aligned}
& |(\Xi x)(t)-(\Xi y)(t)| \\
& \leq \mid \int_{t_{0}}^{K} H_{1}(t, \omega) \varphi_{q}\left(\int_{t_{0}}^{K} H_{2}(\varrho, s) \hbar_{1}(s, x(s)) d s\right) d \omega \\
& -\int_{t_{0}}^{K} H_{1}(t, \omega) \varphi_{q}\left(\int_{t_{0}}^{K} H_{2}(\varrho, s) \hbar_{1}(s, y(s)) d s\right) d \omega \mid \\
& \leq \int_{t_{0}}^{K}\left|H_{1}(t, \omega)\right| \mid \varphi_{q}\left(\int_{t_{0}}^{K} H_{2}(\varrho, s) \hbar_{1}(s, x(s)) d s\right) \\
& -\varphi_{q}\left(\int_{t_{0}}^{K} H_{2}(\omega, s) \hbar_{1}(s, y(s)) d s\right)\left|d \omega \leq(q-1) \lambda^{q-2} \int_{t_{0}}^{K}\right| H_{1}(t, \omega) \mid \\
& \cdot\left(\int_{t_{0}}^{K}\left|H_{2}(\omega, s)\right|\left|\hbar_{1}(s, x(s))-\hbar y_{1}(s, y(s))\right| d s\right) d \omega \\
& \leq \ell(q-1) \lambda^{q-2} \int_{t_{0}}^{K}\left|H_{1}(t, \omega)\right|\left(\int_{t_{0}}^{K}\left|H_{2}(\omega, s)\right||x(s)-y(s)| d s\right) d \omega \\
& \leq \ell(q-1) \lambda^{q-2} \int_{t_{0}}^{K}\left|H_{1}(t, \varpi)\right|\left(\Delta_{2}\|x-y\|\right) d \omega \leq \ell(q-1) \lambda^{q-2} \Delta_{1} \Delta_{2}\|x-y\|,
\end{aligned}
$$

for $x, y \in \mathscr{C}\left[t_{0}, K\right]$. So, $\|\Xi x-\Xi y\| \leq \ell(q-1) \lambda^{q-2} \Delta_{1} \Delta_{2}\|x-y\|$. Hence, in view of the well-known contraction principle due to Banach, we follow that $\Xi$ admits a fixed point uniquely. Thus, the generalized $p$-Laplacian FBVP (4) involves a solution uniquely.

Theorem 14. If hypotheses (HP1) and (HP2) hold, then the generalized p-Laplacian FBVP (4) has a solution such that $\Omega_{2}<1$. Moreover, the set containing solutions of the generalized $p$-Laplacian FBVP (4) is bounded.

Proof. According to Theorem 13, $\Xi$ is Lipschitz and by Proposition $7, \Xi$ is $\xi$-Lipschitz which yields that $\Xi$ is $\xi$-condensing. With the aid of Theorem 8 , we need to prove that

$$
\mathbb{W}=\left\{x \in \mathscr{C}\left[t_{0}, K\right]: \theta \in[0,1] \text { exists so that } x=\theta \Xi(x)\right\} \text {, }
$$

is bounded. For this regard, we suppose that $x \in \mathbb{W}$ for some $\theta \in[0,1]$ and for each $t \in\left[t_{0}, K\right]$. Then, from the growth condition of $\Xi$ derived in Theorem 11, we may write

$$
\|x\|=\|\theta \Xi(x)\| \leq \Omega_{1}+\Omega_{2}\|x\| .
$$

Hence,

$$
\|x\| \leq \frac{\Omega_{1}}{1-\Omega_{2}},
$$

which yields that $\mathbb{W}$ is a bounded set contained in $\mathscr{C}\left[t_{0}, K\right]$. By Theorem 8 , one can understand that $\Xi$ involves at least a fixed point which confirms the existence of at least a solution for the proposed generalized $p$-Laplacian FBVP (4), and hence $\mathbb{W}$ consisting of solutions of the mentioned FBVP (4) is a bounded subset of $\mathscr{C}\left[t_{0}, K\right]$. This ends the proof.

3.2. Analysis of the Stability. In this part, we discuss on four kinds of stability for the generalized $p$-Laplacian FBVP (4) as follows $[6,7]$.

Definition 15. The generalized p-Laplacian FBVP (4) is called Ulam-Hyers stable if there is a real number $\mathfrak{c}_{y_{1}}>0$ such that for every $\kappa>0$ and every solution $\tilde{x} \in \mathscr{C}\left[t_{0}, K\right]$ of the inequality

$$
\left|{ }^{C} D^{\beta, \delta}\left(\varphi_{p}\left({ }^{C} D^{\chi, \rho} \tilde{x}(t)\right)\right)-\hbar_{1}(t, \tilde{x}(t))\right| \leq \kappa,
$$

there is a unique solution $x \in \mathscr{C}\left[t_{0}, K\right]$ of (4) such that

$$
\|x-\tilde{x}\| \leq \mathfrak{E}_{\mathrm{y}_{1}} \kappa,\left(t \in\left[t_{0}, K\right]\right) .
$$

Definition 16. The generalized $p$-Laplacian FBVP (4) is called the generalized Ulam-Hyers stable with respect to $\rho$ $\in \mathscr{C}\left(\mathbb{R}^{>0}, \mathbb{R}^{>0}\right)$ with $\rho(0)=0$, if for each approximate solution $\tilde{x} \in \mathscr{C}\left[t_{0}, K\right]$ of inequality (47), there is a unique solution $x \in \mathscr{C}\left[t_{0}, K\right]$ of $(4)$ so that

$$
\|x-\tilde{x}\| \leq \rho(\kappa),\left(t \in\left[t_{0}, K\right]\right) .
$$


Definition 17. The generalized p-Laplacian FBVP (4) is called Ulam-Hyers-Rassias stable with respect to $\psi \in \mathscr{C}\left[t_{0}\right.$, $K]$ if there is a real number $\boldsymbol{\Omega}_{\hbar_{1}, \psi}>0$ such that for every $\kappa$ $>0$ and approximate solution $\tilde{x} \in \mathscr{C}\left[t_{0}, K\right]$ of the inequality

$$
\left|{ }^{C} D^{\beta, \delta}\left(\varphi_{p}\left({ }^{C} D^{\chi, \rho} \tilde{x}(t)\right)\right)-\hbar_{1}(t, \tilde{x}(t))\right| \leq \psi(t) \kappa,
$$

there is a unique solution $x \in \mathscr{C}\left[t_{0}, K\right]$ of (4) so that

$$
\|x-\tilde{x}\| \leq \mathfrak{\Re}_{\mathrm{y}_{1}, \psi} \kappa \psi(t),\left(t \in\left[t_{0}, K\right]\right) .
$$

Definition 18. The generalized p-Laplacian FBVP (4) is called the generalized Ulam-Hyers-Rassias stable with respect to $\psi \in \mathscr{C}\left[t_{0}, K\right]$, if there is a real number $\mathfrak{\Re}_{y_{1}, \psi}>0$ such that for each approximate solution $\tilde{x} \in \mathscr{C}\left[t_{0}, K\right]$ of inequality (50), there is a unique solution $x \in \mathscr{C}\left[t_{0}, K\right]$ of (4) such that

$$
\|x-\tilde{x}\| \leq \boldsymbol{\Omega}_{\mathrm{y}_{1}, \psi} \psi(t),\left(t \in\left[t_{0}, K\right]\right) .
$$

Remark 19. The function $\tilde{x} \in \mathscr{C}\left[t_{0}, K\right]$ is a solution of (47) if and only if there exists a function $\Psi \in \mathscr{C}\left[t_{0}, K\right]$ such that

$$
\begin{aligned}
& |\Psi(t)| \leq \kappa \text { for } t \in\left[t_{0}, K\right] \\
& { }^{C} D^{\beta, \delta}\left(\varphi_{p}\left({ }^{C} D^{\chi, \rho} \tilde{x}(t)\right)\right)=\hbar_{1}(t, \tilde{x}(t))+\Psi(t) \text { for } t \in\left[t_{0}, K\right]
\end{aligned}
$$

Remark 20. The function $\tilde{x} \in \mathscr{C}\left[t_{0}, K\right]$ is a solution of (50) if and only if there exists a function $\Phi \in \mathscr{C}\left[t_{0}, K\right]$ such that

$$
\begin{aligned}
& |\Phi(t)| \leq \kappa \psi(t) \text { for } t \in\left[t_{0}, K\right] \\
& { }^{C} D^{\beta, \delta}\left(\varphi_{p}\left({ }^{C} D^{\chi, \rho} \tilde{x}(t)\right)\right)=\hbar_{1}(t, \tilde{x}(t))+\Phi(t) \text { for } t \in\left[t_{0}, K\right]
\end{aligned}
$$

Theorem 21. If the hypothesis (HP2) and the inequality (42) are valid, then the unique solution of the generalized p-Laplacian FBVP (4) is Ulam-Hyers stable and is the generalized Ulam-Hyers stable.

Proof. Set $\kappa>0$ and let $\tilde{x} \in \mathscr{C}\left[t_{0}, K\right]$ be the approximate solution of (47) and $x \in \mathscr{C}\left[t_{0}, K\right]$ be the unique solution of the approximate generalized $p$-Laplacian FBVP

$$
\begin{cases}{ }^{C} D^{\beta, \delta}\left(\varphi_{p}\left({ }^{C} D^{\chi, \rho} \tilde{x}(t)\right)\right)=\hbar_{1}(t, \tilde{x}(t))+\Psi(t), & \left(t \in\left[t_{0}, K\right], t_{0} \geq 0\right), \\ \tilde{x}\left(t_{0}\right)+\mu_{1} \tilde{x}(K)=\sigma_{1} \int_{t_{0}}^{K} \hbar_{2}(s) d s, & \left(\mu_{1} \neq-1\right), \\ \tilde{x}^{\prime}\left(t_{0}\right)+\mu_{2} \tilde{x}^{\prime}(K)=\sigma_{2} \int_{t_{0}}^{K} \hbar_{3}(s) d s, & \left(\mu_{2} \neq-\left(\frac{t_{0}}{K}\right)^{\rho-1}\right), \\ { }^{C} D^{\chi, \rho} \tilde{x}\left(t_{0}\right)=0,{ }^{C} D^{\chi, \rho} \tilde{x}(K)=v^{C} D^{\chi, \rho} \tilde{x}(\eta), & \left(\eta \in\left(t_{0}, K\right)\right),\end{cases}
$$

with $v \neq\left(K^{\delta}-t_{0}^{\delta} / \eta^{\delta}-t_{0}^{\delta}\right)^{q-1}$. According to Theorem 10, we get

$$
\begin{aligned}
\tilde{x}(t)= & \int_{t_{0}}^{K}\left(H_{1}(t, \omega) \varphi_{q}\left(\int_{t_{0}}^{K} H_{2}(\omega, s)(\hbar(s, \tilde{x}(s))+\Psi(t)) d s\right)\right. \\
& \left.+\frac{\sigma_{1} \hbar(\omega)}{1+\mu_{1}}+\frac{\sigma_{2} \hbar_{3}(\varpi)}{t_{0}^{\rho-1}+\mu_{2} K^{\rho-1}}\left(\frac{t^{\rho}-t_{0}^{\rho}}{\rho}-\frac{\mu_{1}}{1+\mu_{1}} \frac{K^{\rho}-t_{0}^{\rho}}{\rho}\right)\right) d \omega,
\end{aligned}
$$

where $H_{1}(t, \omega)$ and $H_{2}(t, s)$ are defined by (21) and (22), respectively. Hence, from Theorem 13, we estimate

$$
\begin{aligned}
|x(t)-\tilde{x}(t)| \leq & \mid \int_{t_{0}}^{K} H_{1}(t, \omega) \varphi_{q}\left(\int_{t_{0}}^{K} H_{2}(\varrho, s) \hbar_{1}(s, x(s)) d s\right) d \omega \\
& -\int_{t_{0}}^{v} H_{1}(t, \omega) \varphi_{q}\left(\int_{t_{0}}^{K} H_{2}(\omega, s)\left(\hbar_{1}(s, \tilde{x}(s))+\Psi(t)\right) d s\right) d \omega \mid \\
\leq & \int_{t_{0}}^{K}\left|H_{1}(t, \omega)\right| \mid \varphi_{q}\left(\int_{t_{0}}^{K} H_{2}(\omega, s) \hbar_{1}(s, x(s)) d s\right) \\
& -\varphi_{q}\left(\int_{t_{0}}^{K} H_{2}(\varrho, s) \hbar_{1}(s, \tilde{x}(s)) d s\right) \mid d \omega \\
& +\int_{t_{0}}^{K}\left|H_{1}(t, \varpi)\right| \varphi_{q}\left(\int_{t_{0}}^{K}\left|H_{2}(\varrho, s)\right||\Psi(t)| d s\right) d \omega \\
\leq & \ell(q-1) \lambda^{q-2} \Delta_{1} \Delta_{2}\|x-\tilde{x}\|+\Delta_{1}\left(\Delta_{2} \kappa\right)^{q-1} .
\end{aligned}
$$

Thus,

$$
\|x-\tilde{x}\| \leq \frac{\Delta_{1}\left(\Delta_{2} \kappa\right)^{q-1}}{1-\ell(q-1) \lambda^{q-2} \Delta_{1} \Delta_{2}}=\mathfrak{G}_{\mathrm{y}_{1}} \kappa,
$$

where $\quad \mathfrak{c}_{\mathrm{y}_{1}}:=\Delta_{1} \Delta_{2}^{q-1} \kappa^{q-2} / 1-\ell(q-1) \lambda^{q-2} \Delta_{1} \Delta_{2}$. This shows that the generalized $p$-Laplacian FBVP (4) is UlamHyers stable. Along with this, if $\|x-\tilde{x}\| \leq \rho(\kappa)$ so that $\rho(0)$ $=0$, then the solution related to the generalized $p$-Laplacian FBVP (4) is the generalized Ulam-Hyers stable, and the proof is completed.

Theorem 22. Let the hypothesis (HP2) and (42) are valid, and there exists an increasing function $\psi(t) \in \mathscr{C}\left[t_{0}, K\right]$; there exists $\lambda_{\psi}>0$ such that $\int_{t_{0}}^{K}|\psi(s)| d s \leq \lambda_{\psi} \psi(t), \forall t \in\left[t_{0}, K\right]$. Then, the unique solution of the generalized p-Laplacian FBVP (4) is Ulam-Hyers-Rassias stable and thus is the generalized Ulam-Hyers-Rassias stable.

Proof. Consider $\kappa>0$ and let $\tilde{x} \in \mathscr{C}\left[t_{0}, K\right]$ be the approximate solution of (50) and $x \in \mathscr{C}\left[t_{0}, K\right]$ be the unique solution of the generalized $p$-Laplacian FBVP (4). By remark 20, we have 


$$
\begin{cases}{ }^{C} D^{\beta, \delta}\left(\varphi_{p}\left({ }^{C} D^{\chi, \rho} \tilde{x}(t)\right)\right)=\hbar_{1}(t, \tilde{x}(t))+\Phi(t), & \left(t \in\left[t_{0}, K\right], t_{0} \geq 0\right), \\ \tilde{x}\left(t_{0}\right)+\mu_{1} \tilde{x}(K)=\sigma_{1} \int_{t_{0}}^{K} \hbar_{2}(s) d s, & \left(\mu_{1} \neq-1\right), \\ \tilde{x}^{\prime}\left(t_{0}\right)+\mu_{2} \tilde{x}^{\prime}(K)=\sigma_{2} \int_{t_{0}}^{K} \hbar_{3}(s) d s, & \left(\mu_{2} \neq-\left(\frac{t_{0}}{K}\right)^{\rho-1}\right), \\ { }^{C} D^{\chi, \rho} \tilde{x}\left(t_{0}\right)=0,{ }^{C} D^{\chi, \rho} \tilde{x}(K)=v^{C} D^{\chi, \rho} \tilde{x}(\eta), & \left(\eta \in\left(t_{0}, K\right)\right),\end{cases}
$$

with $v \neq\left(K^{\delta}-t_{0}^{\delta} / \eta^{\delta}-t_{0}^{\delta}\right)^{q-1}$. In view of Theorem 10, we have

$$
\begin{aligned}
\tilde{x}(t)= & \int_{t_{0}}^{K}\left(H_{1}(t, \omega) \varphi_{q}\left(\int_{t_{0}}^{K} H_{2}(\Phi, s)\left(\hbar_{1}(s, \tilde{x}(s))+\Phi(s)\right) d s\right)\right. \\
& \left.+\frac{\sigma_{1} \hbar_{2}(\Phi)}{1+\mu_{1}}+\frac{\sigma_{2} \hbar_{3}(\varpi)}{t_{0}^{\rho-1}+\mu_{2} K^{\rho-1}}\left(\frac{t^{\rho}-t_{0}^{\rho}}{\rho}-\frac{\mu_{1}}{1+\mu_{1}} \frac{K^{\rho}-t_{0}^{\rho}}{\rho}\right)\right) d \Phi,
\end{aligned}
$$

where $H_{1}(t, \omega)$ and $H_{2}(t, s)$ are defined by (21) and (22), respectively. Hence, we can immediately estimate that

$$
\|x-\tilde{x}\| \leq \frac{\Delta_{1}\left(\Delta_{2} \kappa \lambda_{\psi} \psi(t)\right)^{q-1}}{1-\ell(q-1) \lambda^{q-2} \Delta_{1} \Delta_{2}}=\Re_{\hbar, \psi} \kappa \psi(t),
$$

where $\mathfrak{\Re}_{\mathrm{y}_{1}, \psi}:=\Delta_{1} \Delta_{2}^{q-1} \lambda_{\psi}^{q-1}(\kappa \psi(t))^{q-2} / 1-\ell(q-1) \lambda^{q-2} \Delta_{1}$ $\Delta_{2}$. This proves that the generalized $p$-Laplacian FBVP (4) is Ulam-Hyers-Rassias stable. Furthermore, if $\kappa=1$, then the solution of generalized p-Laplacian FBVP (4) is the generalized Ulam-Hyers-Rassias stable, and the proof is completed.

\section{Example}

As an application to validate the theoretical results, an illustrative example is given here.

Example 23. Regarding to the given FBVP (4), we provide a special structure of the generalized problem having the fractional composite p-Laplacian as

$$
\left\{\begin{array}{l}
{ }^{C} D^{1.08,0.5}\left(\varphi_{\frac{5}{4}}\left({ }^{C} D^{1.09,0.7} x(t)\right)\right)=\frac{|6 \sin x(t)|}{|12000 \sin x(t)|+12000}+0.18 e^{t},(t \in[0,1]) \\
x(0)+0.1 x(1)=0.8 \int_{0}^{1}\left(2 e^{3 s}+1\right) d s \\
x^{\prime}(0)+0.2 x^{\prime}(1)=0.9 \int_{0}^{1} \cos (s) d s \\
{ }^{C} D^{1.09,0.7} x(0)=0,{ }^{C} D^{1.09,0.7} x(1)=0.3^{C} D^{1.09,0.7} x(0.25),
\end{array}\right.
$$

in which the following parameters are considered $\beta=$ 1.08, $\chi=1.09, \delta=0.5, \rho=0.7, \mu_{1}=0.1, \mu_{2}=0.2, v=0.3, \sigma_{1}$ $=0.8, \sigma_{2}=0.9, \eta=0.25, \lambda=1.5, p=5 / 4, q=5, t_{0}=0, K=1$ , and $\varphi_{p}(\theta)=|\theta|^{p-2} \theta$. In addition to these, the continuous functions $\mathrm{y}_{1}, \mathrm{y}_{2}$, and $\mathrm{y}_{3}$ are introduced by

$$
\begin{gathered}
\hbar_{1}(t, x(t))=\frac{|6 \sin x(t)|}{|12000 \sin x(t)|+12000}+0.18 e^{t} d, \\
\hbar_{2}(t)=2 e^{3 t}+1, \hbar_{3}(t)=\cos (t),
\end{gathered}
$$

for $t \in[0,1]$. By utilizing some of above data, we get $\Delta_{1} \simeq$ $=3.0899$ and $\Delta_{2} \simeq 6.4094$. On the other side, for any $x, \widehat{x} \in$ $\mathbb{R}$, we can write

$$
\begin{aligned}
& \left|\hbar_{1}(t, x(t))-\hbar_{1}(t, \widehat{x}(t))\right| \\
& \quad \leq\left|\frac{|6 \sin x(t)|}{|12000 \sin x(t)|+12000}-\frac{|6 \sin \widehat{x}(t)|}{|12000 \sin \widehat{x}(t)|+12000}\right| \\
& \quad \leq \frac{6}{12000}\left|\frac{|\sin x(t)|}{|\sin x(t)|+1}-\frac{|\sin \widehat{x}(t)|}{|\sin \widehat{x}(t)|+1}\right| \\
& \quad \leq \frac{6}{12000}|\sin x(t)-\sin \widehat{x}(t)| \leq \frac{6}{12000}|x(t)-\widehat{x}(t)|,
\end{aligned}
$$

where $\ell=6 / 12000=0.0005$ is obtained. Then, since

$$
\ell(q-1) \lambda^{q-2} \Delta_{1} \Delta_{2} \simeq 0.1336797342<1,
$$

thus the conditions of Theorem 13 are satisfied, and so the generalized composite $p$-Laplacian FBVP (60) has a unique solution. On the other side, since all hypotheses of Theorems 21 and 22 hold, we find out that the given generalized composite $p$-Laplacian FBVP (60) is Ulam-Hyers and UlamHyers-Rassias stable and thus is stable of their generalized type.

\section{Conclusion}

Qualitative analysis such as the investigation of the existence, uniqueness, and stability of fractional differential equations is an important and useful task. In this paper, we studied a generalized fractional composite differential equation with $p$-Laplacian operator equipped with threepoint integral boundary value conditions. We used the classical results for this purpose and obtained the relevant Green's function. The existence and uniqueness of solutions were established by means of topological degree theory and Banach contraction principle. Besides, four types of stability in the sense of Ulam-Hyers, Ulam-Hyers-Rassias, and their generalized versions were analyzed. Finally, we provided an illustrative example to validate our results. In the next researches, one can study these qualitative behaviors of solutions for different generalizations of fractional $p$-Laplacian boundary value problems by means of generalized operators with nonsingular kernels such as Caputo-Fabrizio operators or Atangana-Baleanu operators.

\section{Data Availability}

Data sharing not applicable to this article as no datasets were generated or analyzed during the current study. 


\section{Conflicts of Interest}

The authors declare that they have no competing interests.

\section{Authors' Contributions}

The authors declare that the study was realized in collaboration with equal responsibility. All authors read and approved the final manuscript.

\section{Acknowledgments}

The fourth author would like to thank Prince Sultan University for this work through research group Nonlinear Analysis Methods in Applied Mathematics (NAMAM) group number RG-DES-2017-01-17. The first and fifth authors would like to thank Azarbaijan Shahid Madani University. The authors would like to thank dear respected referees for their constructive and helpful comments.

\section{References}

[1] A. Kilbas, H. Srivastava, and J. J. Trujillo, "Theory and applications of fractional differential equations," in North-Holland Mathematics Studies, vol. 204, North-Holland, Amsterdam, 2006.

[2] S. G. Samko, A. A. Kilbas, and O. I. Marichev, Fractional Integrals and Derivatives: Theory and Applications, Gordon and Breach Science Publishers, London, 1993.

[3] S. Etemad, S. K. Ntouyas, and J. Tariboon, "Existence results for three-point boundary value problems for nonlinear fractional differential equations," Journal of Nonlinear Sciences and Applications, vol. 9, no. 5, pp. 2105-2116, 2016.

[4] A. Boutiara, K. Guerbati, and M. Benbachir, "Caputo-Hadamard fractional differential equation with three-point boundary conditions in Banach spaces," AIMS Mathematics, vol. 5, no. 1, pp. 259-272, 2019.

[5] C. Derbazi, Z. Baitiche, M. Benchohra, and A. Cabada, "Initial value problem for nonlinear fractional differential equations with $\psi$-Caputo derivative via monotone iterative technique," Axioms, vol. 9, no. 2, p. 57, 2020.

[6] D. H. Hyers, "On the stability of the linear functional equation," Proceedings of the National Academy of Sciences, vol. 27, no. 4, pp. 222-224, 1941.

[7] S. M. Ulam, A Collection of Mathematical Problems, Interscience publ, New York, 1961.

[8] M. S. Abdo, S. T. M. Thabet, and B. Ahmad, "The existence and Ulam-Hyers stability results for \psi-Hilfer fractional integrodifferential equations," Journal of Pseudo-Differential Operators and Applications, vol. 11, no. 4, pp. 1757-1780, 2020.

[9] A. Zada, J. Alzabut, H. Waheed, and I. L. Popa, “Ulam-Hyers stability of impulsive integrodifferential equations with Riemann-Liouville boundary conditions," Advances in Difference Equations, vol. 2020, no. 1, 2020.

[10] A. Kheiryan and S. Rezapour, "A multi-singular fractional equation and the Hyers-Ulam stability," International Journal of Applied and Computational Mathematics, vol. 6, no. 6, article 155, 2020.

[11] D. Ma, Z. J. du, and W. Ge, "Existence and iteration of monotone positive solutions for multipoint boundary value problem with p-Laplacian operator," Computers \& Mathematcs with Applications, vol. 50, no. 5-6, pp. 729-739, 2005.

[12] M. M. Matar, A. A. Lubbad, and J. Alzabut, "On p-Laplacian boundary value problems involving Caputo-katugampula fractional derivatives," Mathematical Methods in the Applied Sciences, 2020.

[13] H. Khan, C. Tunc, W. Chen, and A. Khan, "Existence theorems and Hyers-Ulam stability for a class of hybrid fractional differential equations with p-Laplacian operator," Journal of Applied Analysis and Computation, vol. 8, no. 4, pp. 12111226, 2018.

[14] H. Jafari, D. Baleanu, H. Khan, R. A. Khan, and A. Khan, "Existence criterion for the solutions of fractional order $\mathrm{p}$ Laplacian boundary value problems," Boundary Value Problems, vol. 2015, no. 1, 2015.

[15] K. Shah and R. A. Khan, "Existence and uniqueness results to a coupled system of fractional order boundary value problems by topological degree theory," Numerical Functional Analysis and Optimization, vol. 37, no. 7, pp. 887-899, 2016.

[16] M. Sher, K. Shah, M. Feckan, and R. A. Khan, "Qualitative analysis of multi-terms fractional order delay differential equations via the topological degree theory," Mathematics, vol. 8, no. 2, p. 218, 2020.

[17] A. Ali, B. Samet, K. Shah, and R. A. Khan, "Existence and stability of solution to a toppled systems of differential equations of non-integer order," Boundary Value Problems, vol. 2017, no. $1,2017$.

[18] H. Khan, W. Chen, A. Khan, T. S. Khan, and Q. M. Al-Madlal, "Hyers-Ulam stability and existence criteria for coupled fractional differential equations involving p-Laplacian operator," Advances in Difference Equations, vol. 2018, no. 1, 2018.

[19] K. Shah and W. Hussain, "Investigating a class of nonlinear fractional differential equations and its Hyers-Ulam stability by means of topological degree theory," Numerical Functional Analysis and Optimization, vol. 40, no. 12, pp. 1355-1372, 2019.

[20] U. N. Katugampola, "A new approach to generalized fractional derivatives," Bulletin of Mathematical Analysis and Applications, vol. 6, pp. 1-15, 2014.

[21] D. Guo, V. Lakshmikantham, and X. Liu, Nonlinear Integral Equations in Abstract Spaces, Mathematics and Its Applications, Kluwer Academic Publishers, Dordrecht, The Netherlands, 1996.

[22] F. Isaia, "On a nonlinear integral equation without compactness," Acta Mathematica Universitatis Comenianae, vol. 75, pp. 233-240, 2006. 